\title{
Korean rheumatology workforce from 1992 to 2015: current status and future demand
}

\author{
Chan Uk Lee, Ji Na Kim, Ji-Won Kim, Sung-Hoon Park, Hwajeong Lee, Seong-Kyu Kim, and \\ Jung-Yoon Choe
}

Division of Rheumatology, Department of Internal Medicine, Catholic University of Daegu School of Medicine, Daegu, Korea

Received: December 13, 2016 Revised : February 2, 2017 Accepted: March 31, 2017

\section{Correspondence to}

Sung-Hoon Park, M.D.

Division of rheumatology, Department of Internal Medicine, Catholic University of Daegu

School of Medicine, 33 Duryugongwon-ro 17-gil, Nam-gu,

Daegu 42472, Korea

Tel: +82-53-650-4027

Fax: +82-53-621-3166

E-mail:youriig9@gmail.com
Background/Aims: Rheumatology in Korea has rapidly advanced in the 24 years since the subspecialty board certification program was established in 1992. The objective of this investigation was to analyze the distribution of rheumatology practices in Korea in order to better understand the rheumatology workforce.

Methods: Using a membership list from the Korean College of Rheumatology (KCR), we obtained information on practicing rheumatologists. We mapped the ratio of rheumatologists to the general population and to patients with rheumatologic disease using data from Statistics Korea and the 2015 Health Insurance Review \& Assessment Service (HIRA).

Results: In the 16 administrative districts of Korea in 2015, there were 311 practicing rheumatologists on the list of KCR members. There were 218 members practicing in metropolitan areas and 93 members in the provinces. The mean number of rheumatologists per 100,000 people was 0.60 , with $0.33 / 100,000$ in the provinces, but $0.92 / 100,000$ in metropolitan areas, a 2.7 -fold difference. The number of rheumatologists per 100,000 patients with chronic rheumatic disease was 17.21 in metropolitan areas but 6.57 in the provinces, according to 2015 HIRA data. This geographic maldistribution emerged as a problem; indeed, the regional disparity in the distribution of Korean rheumatologists was striking when compared to the published medical professional distribution in 2014.

Conclusions: Because of the uneven distribution of rheumatologists, it is likely that some patients with chronic rheumatic conditions have limited access to rheumatology care. Thus, a policy-based approach is needed to alleviate this disparity.

Keywords: Rheumatologists; Workforce; Distribution

\section{INTRODUCTION}

Compared with most other specialties, modern rheumatology in Korea started relatively late, only 36 years ago. Advancements in medications (biologics) and diagnostic tools for rheumatology have made it increasingly difficult for primary care physicians to provide comprehensive rheumatology care due to the growing array of advanced services $[1,2]$. Concerns about demands for rheumatology workforce are also increasing with the growth of the aging population.

Due to the paucity of research on this topic, it is difficult to assess the current status of the rheumatology workforce and predict how the rheumatology workforce will change in Korea. We sought to determine the status and distribution of the rheumatology workforce, since 
the demand for treatment and the number of patients with rheumatic disease is expected to increase.

\section{METHODS}

Using the membership list of the Korean College of Rheumatology (KCR), we obtained the addresses of all practicing rheumatologists for the period from 1992 to 2015. Based on their work addresses, the rheumatologists were divided into three groups according to employment status. Group A included those employed at tertiary hospital. Group B comprised rheumatologists at hospitals offering up to secondary care. Group C was composed of those who worked at clinics focused on outpatient care.

To examine the supply and distribution of the rheumatology workforce, we mapped the ratio of rheumatologists to the population and to patients with rheumatologic disease from 1992 to 2015 using Statistics Korea data and from 2010 to 2015 using Health Insurance Review \& Assessment Service (HIRA) data.

Patients were categorized into seven groups according to the Korean Standard Classification of Disease (KCD), based on medical claims data from the HIRA database for the 16 administrative districts. Because data for the Sejong district were insufficient in 2015, this district was excluded. For a comparison of distributions, the 2014 Annual Report Membership Statistics Korean Medical Association was used. This study contains data opend in Statistics Korea, that can be exempted from review of Institutional Ethical Review Board.

\section{RESULTS}

\section{The number of rheumatologists}

For the first time in 1992, eight members of the KCR were authenticated. The number of rheumatologists has increased by double digits every year since 1997 [3]. In 2015 , a total of 315 rheumatologists were practicing, with 171 rheumatologists (54.3\%) in group A, 74 (23.4\%) in group B, and $66(21 \%)$ in group C. Two rheumatologists worked at pharmaceutical companies, while one rheumatologist worked at a public health center and another worked at an overseas hospital (Fig. 1).
According to the published internist employment data (2014 Annual Report Membership Statistics Korean Medical Association), the proportion of specialists working in the tertiary hospitals was higher in rheumatologists than in internal medicine (Fig. 2) [4]. The number of rheumatologists has more than quadrupled over the past 15 years, from 73 in 2001 to 315 in 2015. For over 20 years, more than 10 rheumatologists have been certified by the KCR annually. This is a rapid increase compared to the roughly 2-fold increase in internists from 6,425 in 2001 to 13,536 in 2014, and the approximate 2-fold increase in orthopedists from 2,714 in 2001 to 5,482 in 2014, as reported by the Annual Report Membership Statistics Korean Medical Association. Rheumatologists account-

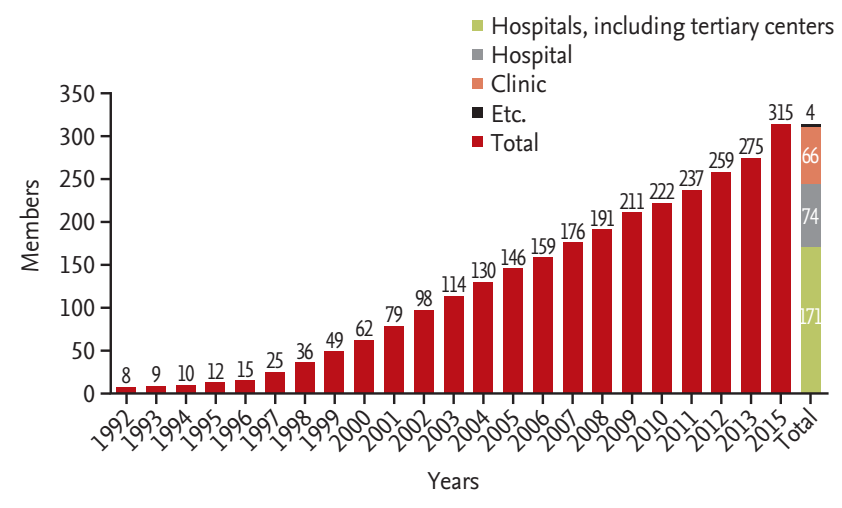

Figure 1. Growth trend for rheumatologist. After complete training program in centers, rheumatologist has been practicing in each medical institution. From the year of 1997, at least 10 members have been increased annually.

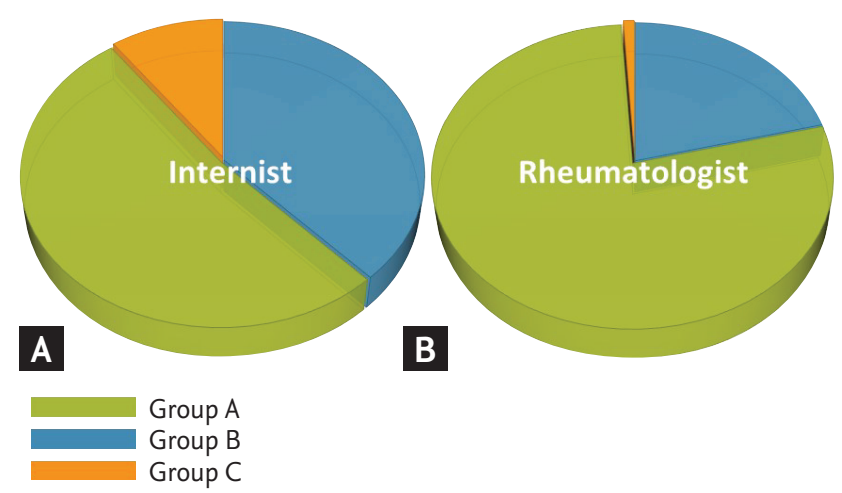

Figure 2. (A). Internist employment data (2014 published 'Annual report membership statistics Korean Medical Association').(B). Rheumatologists working in medical institutes (Group A: hospitals, including tertiary centers; Group B: hospitals offering up to secondary care; Group C: outpatient care). 
ed for $2.3 \%$ of all internists in 2014, compared to just $1.2 \%$ in 2001 . The number of orthopedists was 38.5 -fold that of rheumatologists in 2011, but was only 17.5 -fold that of rheumatologists in 2014 (Fig. 3).

Despite the rapid increase in the number of rheumatologists in Korea, the number remains modest compared to the numbers in several foreign countries $[5,6]$. The number of rheumatologist per 100,000 people is 2.9 in Japan, which is five times that of Korea. Taiwan has a smaller ratio of medical doctors to the general population than Korea, but has 1.31 rheumatologists per 100,000 people, twice the number in Korea. In an exceptional case, China has a shortage compared to South Korea, with 0.17 rheumatologists per 100,000 [7]. Even when compared with the United Kingdom, the United States, and Canada [8], which have advanced primary medical systems and large numbers of medical doctors, Korea still has a relative shortage of rheumatologists (Fig. 4).

\section{Regional distribution of rheumatologists}

Fig. 5 depicts the 311 rheumatologists based on work-

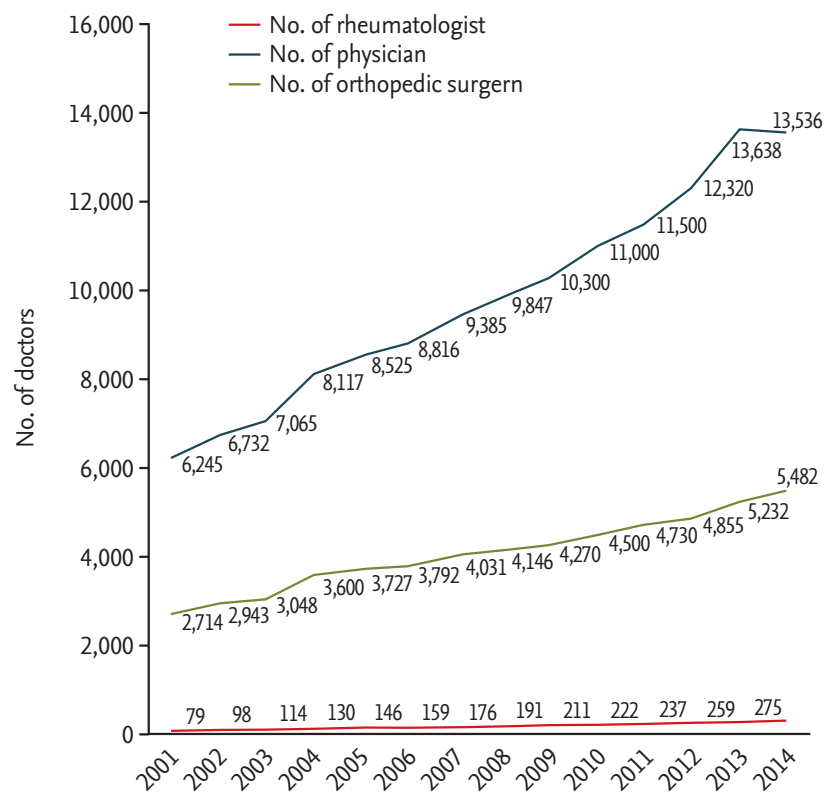

Figure 3. Korean rheumatologist: the past and present. In the past 15 years (2001 to 2014), rheumatologist increased four times over; however, physician and orthopedic surgeon has more doubled over. The share of rheumatologist in physician has been grown-up from $1.2 \%$ in 2001 to $2.3 \%$ in 2014 . place address in 2015. Rheumatologists were concentrated in seven metropolitan cities along the axis of the Gyeongbu line and the Honam line. More than half of the rheumatologists worked in the zone straddling Seoul, Incheon, and Gyeonggi-do. Two hundred and fifty-two specialists were active in metropolitan areas with populations exceeding 1 million people, and 59 specialists were active in administrative areas with low population densities.

Fig. 5 also illustrates the population by administrative district, number of rheumatologists, and number of rheumatologists per 100,000 people based on data from the Population and Housing Census of 2015. Administrative districts marked in red are metropolitan areas, each with a population density of over 50 million people per square kilometer. In metropolitan areas, the number of rheumatologists per 100,000 people exceeded the national average of 0.54 . The number of rheumatologists per 100,000 residents was 1.23 in Seoul, 0.78 in Daejeon, 0.93 in Gwangju, and 0.88 in Daegu, whereas the number was 0.1 in Chungcheongnam-do, 0.1 in Jeollanam-do, and 0.19 in Gyeongsangbuk-do. The sum of the general population of four provinces (Jeollanam-do, Chungcheongnam-do, Gyeongsangbuk-do, and Gyeongsangnam-do) was similar to that of Seoul, but the sum of the number of rheumatologists was 5 -fold smaller.

There was a clear disparity in the difference in distribution of rheumatology workforce not only by administrative district but also by living areas. Living areas mean adjacent administrative area which shares

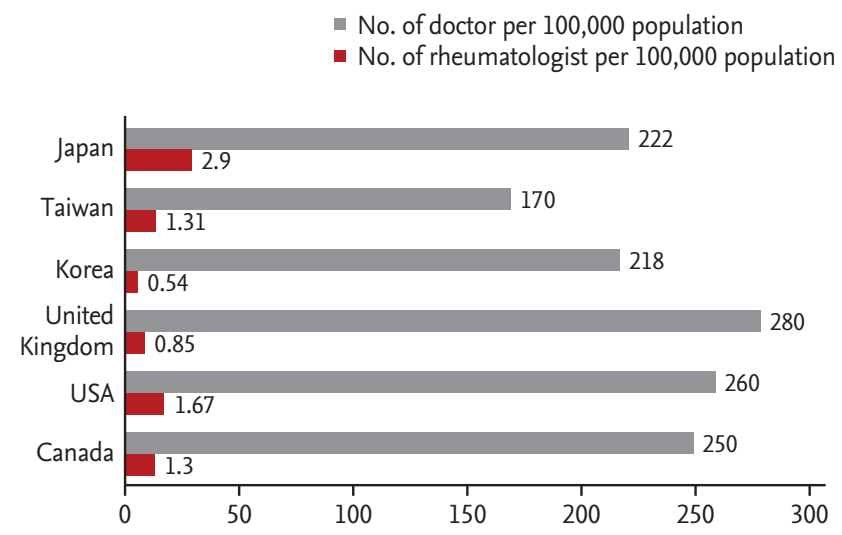

Figure 4. The number of rheumatologist in Korea is in shortage, compared to other countries, despite fast increase in the number of rheumatologist. 
economic and cultural [9]. Annually, HIRA divides the country into seven areas (A: Seoul area; B: Gyeonggi area; C: Gangwon area; D: Chungcheong area; E: Jeolla area; F: Gyeongsang area; G: Jeju area) and publishes regional data of medical centers institutions by region $[10,11]$, we applied these seven areas to regional distribution of rheumatologist and other medical parts. In Gangwon area, there were five rheumatologists and the number of rheumatologist per 100,000 people (0.35) was lower than in any other evaluated region in Korea. The number of rheumatologists per 100,000 people was 1.23 in Seoul area, which was 3-fold over than the number in Gangwon. In Seoul areas, there were 4,079 internists as of 2014, and the number of internists per 100,000 people (40.3) was higher than in any other evaluated region in Korea. The number of internists per 100,000 people was 16.7 in Gyeonggi area, which was 2-fold lower than the number in Seoul area. The distribution of orthopedists was similar to the distribution of internists. The number of orthopedists was 14.2 per 100,000 people in Seoul area and 8.9 per 100,000 people in Gyeonggi area. The distribution of physiatrist looked equally comparable with other medical parts (Fig. 6).

Such regional differences were also found in the number of rheumatologists relative to the number of patients with rheumatic disease. The number of patients in seven categories of rheumatic disease was extracted from the total number of patients in the respective regions where reimbursement claims were filed with the HIRA service of the Health Insurance Corporation in 2015. The number of patients in Seoul was 826,821, the largest nationwide, according to the HIRA data. The number of rheumatologists per 100,000 patients with rheumatic disease was 12.09 in Seoul, which was 2-fold higher than the country-wide average of 6.64 and 3-fold higher than the provincial average of 3.78 .

The number of patients in Chungcheongnam-do was 177,498 , the 9th largest nationwide. However, the number of doctors per 100,000 rheumatic patients was 1.12, the lowest across the country and 10 times lower than the number in Seoul. A significant difference was found by province. The number of rheumatologists per 100,000 patients with rheumatic disease was 6.96 in Jeollabuk-do, which was more than twice the number in

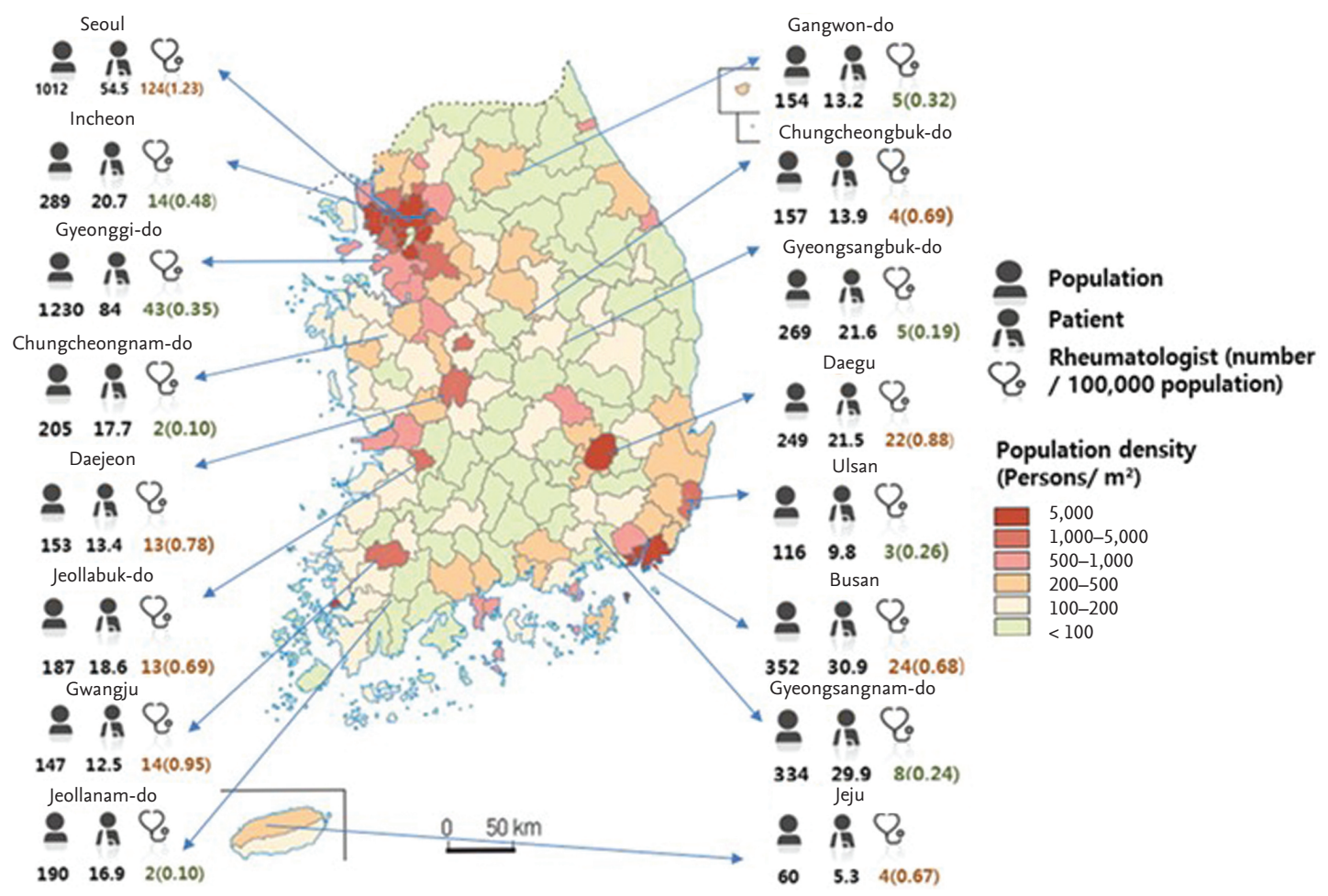

Figure 5. Number of rheumatologist and physician per population, patients in each administrative districts. On map, each color scale implies degree of population density and the far red areas are metropolitan cities with high population density in 2015 (Seoul, Busan, Incheon, Daegu, Gwangju, Daejeon, and Ulsan). 
Gyeongsangbuk-do (2.30) or Gyeongsangnam-do (2.66) (Table 1).

\section{DISCUSSION}

The number of patients in seven categories of rheumatic disease (extracted from HIRA data) increased from 1.6 million in 2010 to 2.6 million in 2015. Moreover, national insurance service costs related to those patients soared from KRW 680 billion to KRW 980 billion in the same period (Fig. 7). Such sharp increases are likely attributable to the growth of the aging population, as well as an increase in the number of patients who have been newly diagnosed with diseases due to greater awareness of rheumatologic disease and improvement of access to rheumatologic care, due to greater awareness of rheumatologic disease [12]. The number of patients and medical expenditures are expected to increase further, and there will be a corresponding increasing demand on the medical workforce related to rheumatic disease in the future. It is difficult to accurately predict the future demand for rheumatologists. According to estimates from a 2003 National Health Interview Survey of 31,000 adults in the United States, by 2030, about 67 million adults will have doctor-diagnosed, self-reported arthritis, and 25 million will have arthritis-attributable activity limitations [13]. As a result, healthcare costs are predicted to double, and the number of rheumatologic patients is expected to grow, leading to a shortage of rheumatologists. In Canadian research, an ongoing need to train rheumatologists has been highlighted, given that onethirds of the workforce will be retiring in the next 10 years [14]. According to research in the United States, since 2002 the number of doctors of rheumatology increases as increasing of the number of patients with rheumatic disease and medical expenditure, but as the number of retired rheumatologist increases after 2016, the total number of rheumatologists has been predicted to decrease [12]. When compared with other countries, the number of doctors per 100,000 population did not differ greatly, but the number of rheumatologist per
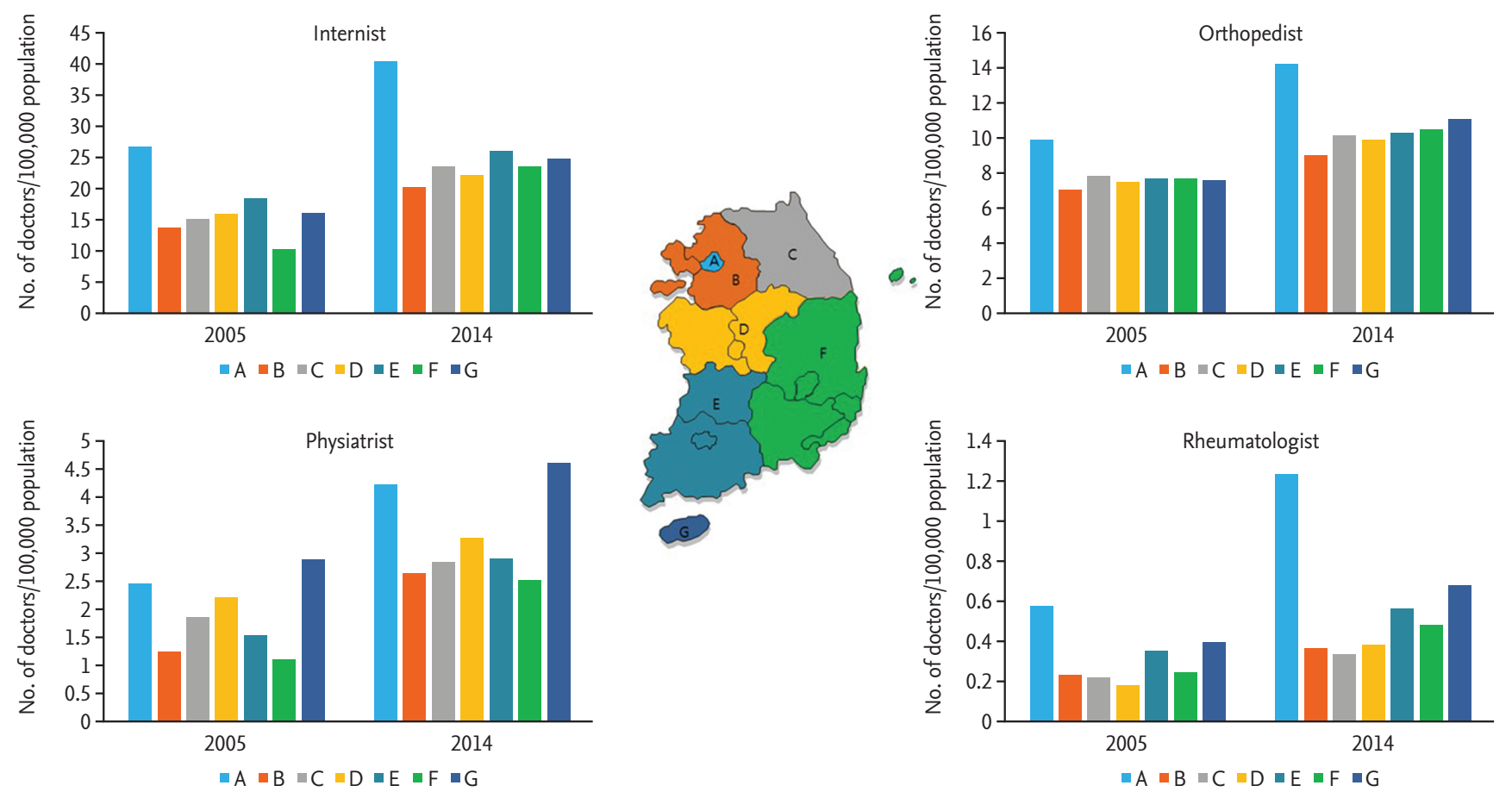

Figure 6. The 2005 and 2014 published 'Annual report membership statistics Korean Medical Association.' In 2014, at internists, orthopedist, physiatrist to populations ratio of seven living areas (A: Seoul area; B: Gyeonggi area; C: Gangwon area; D: Chungcheong area; E: Jeolla area; F: Gyeongsang area; G: Jeju area), difference of regional distribution had been eased compared to 2005 . 


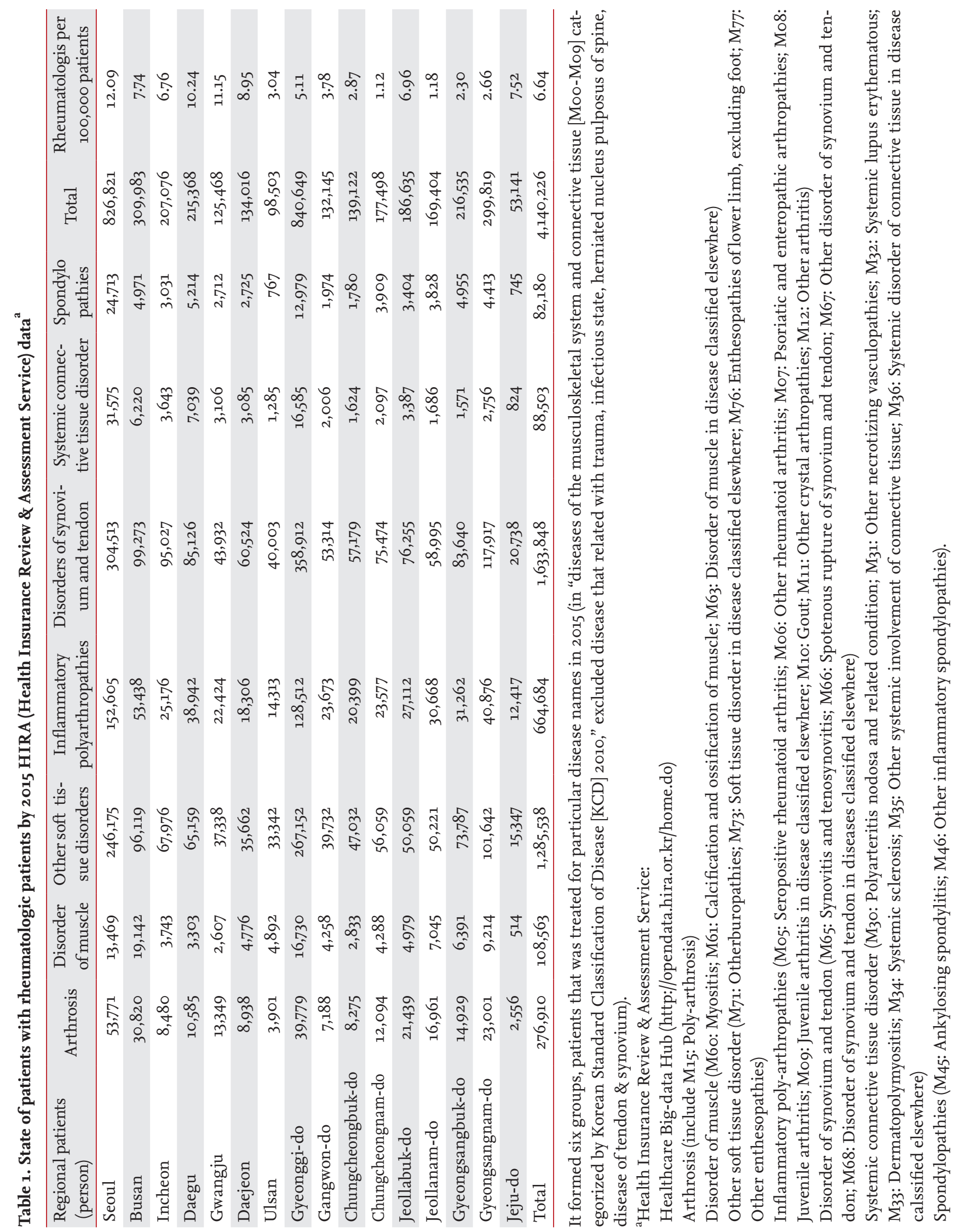




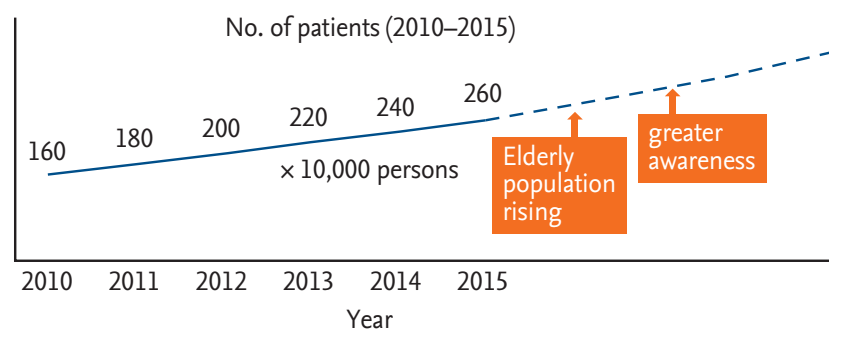

National Health Insurance Service Charge (2010-2015)

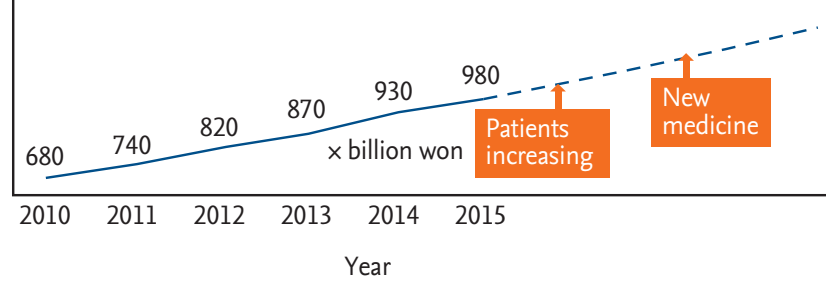

Figure 7. Number of patients (seven groups of Table 1) and National Health Insurance Service Charge from 2010 to 2015. This graphs reveals rising in the number of patients and increasing health expenditures annually (based on 2015 Health Insurance Review \& Assessment Service data).

100,000 populations in Korea was only about one-thirds compared with Canada and the United States. And Korea is entering a super aged society, and the rate of increase in the proportion of elderly population is faster than in the United States. The number of doctors per 100,000 populations has been similar to major OECD countries in Korea, but the number of rheumatologist is still less than in other countries. Since the aging population of Korea is projected to grow rapidly in the future, the demand for rheumatologists is expected to increase, due to the increase in patients with arthritis [3].

Korean rheumatology has achieved fast growth despite being established in less than three decades ago. The number of rheumatologists has increased, but remains inadequate compared to the numbers in other countries. The disparity in regional distribution is more evident in rare condition like pediatric rheumatology. In the United States, 19 states (38\%) have only one or two pediatric rheumatologists, and six states do not have any practicing pediatric rheumatologist. There were many factors to influence this situation, for example, acceptable income, job security, opportunities for teaching and research [15]. Reducing disease activity through more effective treatment would reduce the need for follow-up visits, as would adopting more advanced appointment scheduling practices for established patients [16]. The lower the accessibility to the rheumatologists, the more patients will be concentrated in a nearby big center that are relatively easy to access, resulting in an increase in socioeconomic costs. Therefore, for efficient redistribution of medical resources and proper medical treatment, the larger number of rheumatologists need to practice outside the metropolitan area.

Considering the current situation, a rising demand for rheumatologists is expected due to the increase in the aging population and the increased awareness of rheumatologic disease, which will result in steady increases in the number of patients and medical expenditures. According to a study on the medical preference of cancer and cardiovascular surgery patients, patients prefer medical institutions in Seoul because they perceive that the medical treatment is superior, and benefit from the convenient transportation system such as the Korea Train Express [17,18]. The same phenomenon also appeared in patients with rheumatic diseases and concentration of practitioners in metropolitan areas, particularly Seoul, will likely increase further.

In-depth policy discussion is needed to determine a solution to the maldistribution of rheumatologists. With the aging of the population and the development of medical knowledge, rheumatologic disease will only increase, contributing to an increasing demand for rheumatologists [12,19,20].

Our study focused on the current state of rheumatologists in Korea, the shortage of rheumatologists, and the persistence of the geographic maldistribution over time like another country [21] or medical departments $[22,23]$. Policy discussions attempting to solve the rheumatologic workforce problem must focus not only on gross numbers, but also on the geographic distribution of rheumatologists.

\section{KEY MESSAGE}

1. Rheumatology in Korea has rapidly advanced in the 24 years since the subspecialty board certification program was established.

2. However, the geographic maldistribution of adult rheumatologists is evident in the year of 2015, compared to internists and orthopedists. 
3. For the proper management of chronic, rare rheumatic diseases, a policy-based approach to reduce the concentration of rheumatologists to metropolitan area will be needed.

\section{Conflict of interest}

No potential conflict of interest relevant to this article was reported.

\section{Acknowledgments}

This work was supported by the grant of Research Institute of Medical Science, Catholic University of Daegu (2016).

\section{REFERENCES}

1. Mocsai A, Kovacs L, Gergely P. What is the future of targeted therapy in rheumatology: biologics or small molecules? BMC Med 2014;12:43.

2. Kahlenberg JM, Fox DA. Advances in the medical treatment of rheumatoid arthritis. Hand Clin 2011;27:11-20.

3. Kim HY, Song YW. The dynamic evolution of rheumatology in Korea. Nat Rev Rheumatol 2016;12:183-189.

4. Research Institute for Healthcare Policy, Korean Medical Association. 2014 Annual Report Membership Statistics Korean Medical Association. Seoul (KR): Research Institute for Healthcare Policy, Korean Medical Association, 2015 .

5. Ito S, Gross WL, Reinhold-Keller E, et al. Rheumatology in Japan, Germany, and Egypt: a comparison of medical practices. Acta Med Biol (Niigata) 2006;54:51-58.

6. Organization for Economic Co-operation and Development. OECD Health Statistics 2015 [Internet]. Paris: OECD, 2015 [cited 2017 Sep 27]. Available from: https:// www.oecd.org/els/health-systems/Country-Note-KOREAOECD-Health-Statistics-2015.pdf.

7. Zhang FC. Rheumatology in China: brief introduction of the development of Chinese rheumatology. Rheumatology (Oxford) 2006;45:1323-1324.

8. Brophy J, Marshall DA, Badley EM, et al. Measuring the rheumatology workforce in Canada: a literature review. J Rheumatol 2016;43:1121-1129.

9. Golle K, Granacher U, Hoffmann M, Wick D, Muehlbauer T. Effect of living area and sports club participation on physical fitness in children: a 4 year longitudinal study. BMC Public Health 2014;14:499.

10. Health Insurance Review \& Assessment Service. Gastric cancer adequacy assessment result, 2016 [Internet]. Wonju (KR): HIRA, c2013 [cited 2017 Sep 27]. Available from: http://www.hira.or.kr/re/diag/getDiagEvlList.do?pgmid=HIRAA030004000000.

11. Park J, Lim S, Yim E, Kim Y, Chung W. Factors associated with poor glycemic control among patients with type 2 diabetes mellitus: the fifth Korea National Health and Nutrition Examination Survey (2010-2012). Health Policy Manag 2016;26:125-134.

12. Al Maini M, Adelowo F, Al Saleh J, et al. The global challenges and opportunities in the practice of rheumatology: white paper by the World Forum on Rheumatic and Musculoskeletal Diseases. Clin Rheumatol 2015;34:819829.

13. Hootman JM, Helmick CG. Projections of US prevalence of arthritis and associated activity limitations. Arthritis Rheum 2006;54:226-229.

14. Hooker RS. The extension of rheumatology services with physician assistants and nurse practitioners. Best Pract Res Clin Rheumatol 2008;22:523-533.

15. Henrickson M. Policy challenges for the pediatric rheumatology workforce. Part II. Health care system delivery and workforce supply. Pediatr Rheumatol Online J 2011;9:24.

16. Pincus T, Gibofsky A, Weinblatt ME. Urgent care and tight control of rheumatoid arthritis as in diabetes and hypertension: better treatments but a shortage of rheumatologists. Arthritis Rheum 2002;46:851-854.

17. Jeong HS, Lee KS, Chee HK, Ahn HM, Sim SB. Thoracic and cardiovascular surgeons' perception of the concentration of cardiovascular operations in Seoul metropolitan area's hospitals. Korean J Thorac Cardiovasc Surg 2016;49(Suppl 1):S53-S59.

18. Choi YH. Analysis of user preferences for regional healthcare services. Korean Assoc Public Adm 2001;11(Suppl):139-155.

19. Woolf AD, Pfleger B. Burden of major musculoskeletal conditions. Bull World Health Organ 2003;81:646-656.

20. Desjardins C, St Clair EW, Ehrenberg RG. Analysis of the workforce and workplace for rheumatology and the research activities of rheumatologists early in their careers. Arthritis Rheum 2010;62:3528-3536.

21. American College of Rheumatology Committee on Rheu- 
matology Training and Workforce Issues, FitzGerald JD, Battistone M, et al. Regional distribution of adult rheumatologists. Arthritis Rheum 2013;65:3017-3025.

22. Aneja S, Ross JS, Wang Y, et al. US cardiologist workforce from 1995 to 2007: modest growth, lasting geographic maldistribution especially in rural areas. Health Aff(Mill- wood) 2011;30:2301-2309.

23. Cho CK, Kim DK, Park HJ. Current supply and future workforce projections of anesthesiologists for safe anesthetic care of the Korean population. Anesth Pain Med 2016;11:85-90. 